\title{
Research Paper: Surgical Treatment Outcome in Young Adults With Femoral Neck Fractures
}

\author{
Karim Pisoudeh $^{1}$ (D, Kaveh Gharanizadeh $^{1}$ (D, Mohammad Reza Sarshar $^{1}$ (D, Mani Mahmoudi ${ }^{*}$ (D)
}

1. Bone and Joint Reconstruction Research Center, Shafa Orthopedic Hospital, Iran University of Medical Sciences, Tehran, Iran.

Citation Pisoudeh K, Gharanizadeh K, Sarshar MR, Mahmoudi M. Surgical Treatment Outcome in Young Adults With Femoral Neck Fractures. Journal of Research in Orthopedic Science. 2020; 7(1):23-28. http://dx.doi.org/10.32598/JROSJ.7.1.23

http://dx.doi.org/10.32598/JROSJ.7.1.23

\section{(c) (i) (5)}

Article info:

Received: 10 Jun 2019

Revised: 02 Jul 2019

Accepted: 28 Nov 2019

Available Online: 01 Feb 2020
Keywords:

Femoral neck fracture, Harris hip score,

\section{A B S T RA C T}

Background: Femoral Neck Fracture (FNF) is prevalent in young people. It is mostly due to high-energy trauma and creates many challenges in surgical repair. A few patients with hip fractures can fully recover from the injury and act independently in their daily activities. In this study, we evaluated the results of FNF operation in patients younger than 60 years.

Objectives: This study was conducted to evaluate the outcome of femoral neck surgery in young patients and comparison the complications according to types of surgery ,age,delay and type of FNF fracture.

Methods: This study was a retrospective-prospective descriptive and analytical study on patients 15 to 60 years old with FNF from 2013 to 2017. The surgical efficacy and its results were evaluated using the Harris Hip Scale (HHS). The obtained data were analyzed in SPSS version V. 24

Results: The Mean \pm SD age of 53 study patients was $42.07 \pm 12.5$ years. The Mean \pm SD femoral neck shortening was $7.05 \pm 5.42 \mathrm{~mm}$, and the HHS score was 82.7 \pm 6.9 . Avascular Necrosis $(\mathrm{AVN})$ was seen in $10(18.9 \%)$, malunion in $11(20.8 \%)$, nonunion in $1(1.9 \%)$ and failure in 3 $(5.7 \%)$ patients. Eight patients had reoperation procedures. The type of fracture, delay in surgery, type of operation, and the shortening of the femoral neck were predictive factors of postoperative complications $(\mathrm{P}<0.05)$.

Conclusion: According to this study, reducing the delay for surgery, increasing anatomic reduction, and preventing the shortening of the femoral neck can improve the quality of life, reduce complications, and decrease the economic burden.

\footnotetext{
* Corresponding Author:

Mani Mahmoudi, MD.

Address: Bone and Joint Reconstruction Research Center, Shafa Orthopedic Hospital, Iran University of Medical Sciences, Tehran, Iran

Phone: +98 (912) 2270494

E-mail:dr.mani.mahmoudi@gmail.com
} 


\section{Introduction}

F

emoral Neck Fracture (FNF) is one of the most common injuries among the elderly population and has a high economic burden [1]. Recent studies have shown that the prevalence of hip fractures in Iran and its financial burden is lower compared with the developed countries. The cost of hip fracture fixation in Iran is $0.85 \%$ and $12.4 \%$ of the costs in the world and Middle Eastern countries, respectively [2].

FNF in patients younger than 60 years is usually due to high-energy trauma and creates many challenges in surgical repair. A few patients can return to the activity level before the injury [3]. Based on the femoral head and neck specific circulatory system, as well as the anatomic and functional structure, FNF treatment is very challenging. A safe and appropriate treatment method is necessary to reduce postoperative complications. The goal of FNF treatment is different in elderly and young people. Several studies have been conducted to examine the proposed therapeutic choices for FNF with inconclusive results regarding the selection of proper treatment methods $[4,5]$.

FNF is prevalent in young people. Because of high physical activity in these patients, the treatment choices are limited to FNF fixation. On the other hand, the secondary side effects of surgical treatment can have adverse and irreversible effects on patients' life. Consequently, this study was conducted to evaluate the outcome of femoral neck surgery in young patients.

\section{Methods}

The present study was a retrospective-prospective descriptive-analytical study on the treatment of patients aged 15 to 60 years with FNF from 2013 to 2017. The study population was the patients with FNF referred to the orthopedic surgery center of Firoozgar Hospital of Iran University of Medical Sciences, Tehran, Iran.

All enrolled patients were in the age group of 15 to 60 years, which could visit at the specified times and to follow the treatment at least for 6 months. The exclusion criteria were mental or physical disability, malignancy, pathologic fracture, and follow up less than 6 months.

In the retrospective phase, the patients were evaluated through clinical records for demographic data, type of fracture based on Garden classification, the exact surgical date, number of postoperative visits, time from the last operation, radiography, or magnetic resonance imaging for the presence of malunion, nonunion, or avascular necrosis.

Patients evaluated at 2 weeks, 6 weeks, 3 months, 6 months, and one year after surgery and were asked to be visited annually until the end of the study. In the prospective phase, the candidate patients who discharged from the hospital were asked to join the research and after receiving postoperative recommendations, requested to be visited 2 weeks, 6 weeks, 3 months, 6 months, and one year after the operation, then the annual visits for examination. The clinical outcomes were evaluated by recording the Hip Harris Score (HHS), which included an estimation of the pain, function, range of hip motion, and other abnormalities.

The Chi-square non-parametric test and Fisher exact test were used to compare qualitative variables, and the Independent $\mathrm{t}$ test was used for quantitative variables. The obtained data were analyzed in SPSS V. 24. The significance level was considered 0.05 .

\section{Results}

In our study, 53 patients were enrolled after taking into consideration the inclusion and exclusion criteria. The Mean $\pm \mathrm{SD}$ age of the patients was $42.07 \pm 12.5$ years, and their age range was $20-59$ years. The number of male and female patients were 11 and 42, respectively. The most frequent FNF based on Garden classification was type III, with $56.6 \%$ of all patients (Figure 1). The Mean \pm SD delay in operation was $31.9 \pm 25.7$ hours.

Eighteen (34\%) patients had anatomic fixation and 35 (66\%) non-anatomical fixation. The treatment types in the study patients were as follows: 44 patients $(83 \%)$ with cannulated screw in parallel; $8(15.1 \%)$ with the triangular configuration; and in 1(1.9\%) Dynamic Hip Screw (DHS). Three methods were used in reoperation procedures: screw removal, Total Hip Arthroplasty (THA), and bipolar hemiarthroplasty. The Mean \pm SD durations from the first operation to the reoperation were $154 \pm 80.75$ days (range: $5-450$ days), $320 \pm 340.25$ days (range: 11-630 days), and 12 days, respectively. The Mean \pm SD follow-up for patients was $9.5 \pm 6.9$ months, with a range of 2-24 months. The Mean \pm SD length of the femoral neck shortening was $7.05 \pm 5.42 \mathrm{~mm}$. Twenty-eight (52.8\%) patients had no complication, but 10 (18.9\%) developed Avascular Necrosis (AVN), 11 (20.8\%) malunion, 1 (1.9\%) nonunion and $3(5.7 \%)$ patients had treatment failure (Figure 2). 


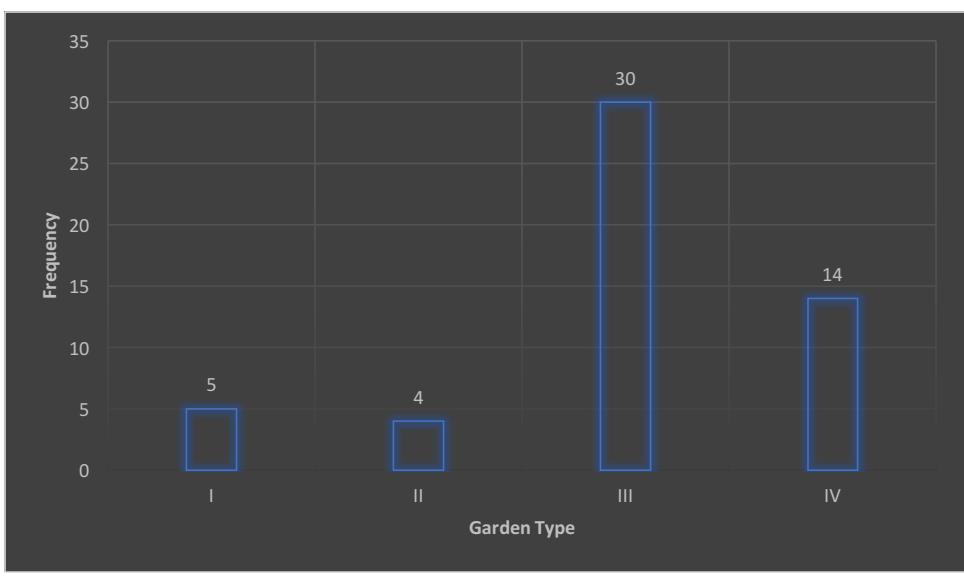

Figure 1. Frequency of type of FNF based on Garden classification

Orthopedic Science

The number of patients undergoing reoperation was 8 (15.1\%). Of them, $7(13.2 \%)$ had with screw removal re-operation, $4(3.5 \%)$ cases had THA, 1 (1.9\%) bipolar, $2(3.8 \%)$ cases with femoral shaft fracture. The

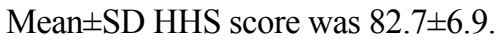

No significant difference was found between age and sex with reoperation and the ensuing complications ( $\mathrm{P}>0.05)$; age and sex were not contributing factors in the increased frequency of reoperation and complications. There was no significant difference between the mean score of HHS in the groups with and without reoperation procedure $(\mathrm{P}=0.6)$. As a result, the HHS score is not a predictor in the frequency of reoperation. There was a significant difference between the frequency of the type of fixation and the rate of postoperative complications $(\mathrm{P}<0.05)$.

The mean age of patients with AVN was significantly lower than those without AVN $(\mathrm{P}<0.001)$ but was considerably higher in patients with malunion $(\mathrm{P}<0.001)$. However, there was no significant difference in the mean age of patients with nonunion and treatment failure $(\mathrm{P}=0.21$ and $\mathrm{P}=0.46$, respectively). There was no significant difference between the mean HHS and complications $(\mathrm{P}=0.45)$. As a result, the HHS average was not the determining factor in postoperative complications. There was a significant difference between the mean length of femoral neck shortening and postoperative complications; therefore, the postoperative complications were determinant. The more femoral neck length decreases, the higher the frequency of postoperative complications $(\mathrm{P}=0.004)$. However, it was not an effective factor in the rate of reoperation $(\mathrm{P}=0.99)$ (Table 1$)$.

The frequency of surgical complications in patients whose operation had been delayed for more than 6 hours was significantly higher than in patients with less than 6 hours of delay in operation $(\mathrm{P}<0.05)$.

There was a significant difference between the type of fracture and the postoperative complications $(\mathrm{P}=0.05)$. The frequency of AVN (51.33, 95\% CI: 13.42-103.77, $\mathrm{P}<0.001$ ), malunion (OR: 34.53, 95\% CI: 12.88-54.33,

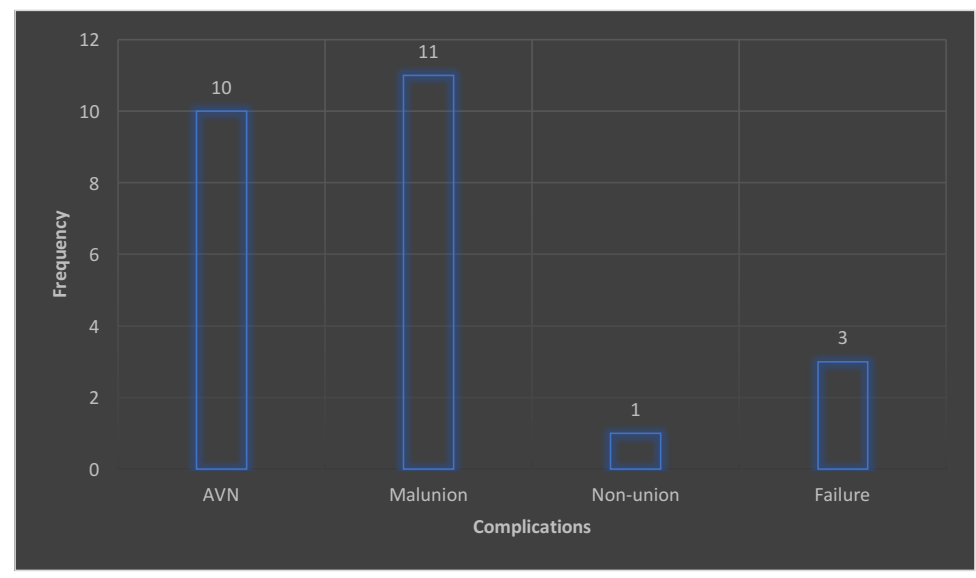

Figure 2. Frequency of complications after the femoral neck operation 
Table 1. The Mean $\pm S D$ values of quantitative variables based on reoperation and postoperative complications

\begin{tabular}{|c|c|c|c|c|c|c|c|}
\hline \multirow{2}{*}{ Variables } & \multicolumn{2}{|c|}{ Reoperation } & \multicolumn{5}{|c|}{ Postoperative Complications } \\
\hline & Yes & No & No Complication & AVN & Malunion & Nonunion & Failure \\
\hline Age (y) & $41.16 \pm 13.6$ & $42.34 \pm 12.3$ & $39.06 \pm 12.34$ & $42.8 \pm 12.56$ & $48.8 \pm 9.3$ & 55 & $41(19.9)$ \\
\hline Delay time in surgical onset (hr) & $16.6 \pm 5.1$ & $28.3 \pm 35.8$ & $29.9 \pm 37.56$ & $14.5 \pm 5.52$ & $13.8 \pm 12.2$ & 90 & $36(37.26)$ \\
\hline HHS & $83.5 \pm 7.7$ & $82.5 \pm 6.8$ & $81.6 \pm 6.78$ & $84.6 \pm 8.81$ & $85 \pm 6.69$ & 75 & 83 \\
\hline Shortening of femoral neck (mm) & $6.8 \pm 6.2$ & $7.09 \pm 5.39$ & $5.2 \pm 4.4$ & $5 \pm 7.07$ & $10 \pm 4.7$ & 20 & - \\
\hline
\end{tabular}

$\mathrm{P}<0.001$ ) and failure (OR: 20.87, 95\% CI: 11.25-23.07, $\mathrm{P}=0.003$ ) in patients with type 3 and 4 fractures is significantly higher. As a result, patients with type 3 and type 4 fractures will be more likely to develop AVN and Malunion and nonunion in one case was reported as a type III.

\section{Discussion}

This study evaluated the results of FNF operation in patients under 60 years old, as well as their complete treatment, complications, and reoperation. No significant difference was noticed between the age, sex, HHS, type of screw, and the frequency of postoperative complications $(\mathrm{P}<0.05)$. Delay in surgery increases postoperative complications. There was a significant difference between the type of fracture and the quality of reduction in the frequency of postoperative complications $(\mathrm{P}<0.05)$. As a result, these four factors determine the treatment result.

Sprague et al., by reviewing 42 articles, evaluated the results of FNF surgery in patients under the age of 60 years. This study reported that malreduction was associated with fracture healing complications, and outcomes in the young FNF studies are similar to elderly populations [6].

Wang et al. published (2018) the therapeutic effect of internal fixation on displaced FNF in middle-aged patients and reported the predictors of successful internal fixation of 117 patients. The patients were 50-60 years old that underwent closed reduction and internal fixation with a cannulated screw. The results of this study showed that the frequency of postoperative complications is high $(84.1 \%)$, and $22 \%$ of patients with favorable reduction had acceptable therapeutic outcomes. While patients with undesirable reduction had complications. The satisfactory therapeutic result was positively correlated with Pauwels' type II fracture (OR: 8.67, $\mathrm{P}=0.025)$ and negatively associated with high alcohol intake $(\mathrm{P}=0.045)$.
The overall findings of this study showed that complications are high for displaced FNF in young patients. In the case of internal fixation, patient selection is crucial. For example, patients with high alcohol intake should not be selected for this treatment, as well as internal fixation, have more successful outcomes in patients with Pauls' type II than type III [7].

In our study, $83 \%$ of cases with type III and IV fracture had complications. However, the complications were significantly higher in the undesired reduction group $(\mathrm{P}=0.05)$. In our study, the optimal therapeutic outcome had a positive correlation with type I-II fracture (OR: 1.33, 95\% CI: $0.75-2.34, \mathrm{P}=0.023$ ) and the effect of high alcohol intake on the outcome of treatment was not investigated.

A retrospective study reported 21 war-injured patients with FNF (from 2001 to 2013). In $56.9 \%$ of patients, the reduction and initial fixation were done within 24 hours, in $57.1 \%$ fixation performed by multiple screws, and $23.8 \%$ were treated by DHS. They reported one case of nonunion, one delayed union, and three AVN cases. No relationship was found between the time of fixation and the type of reduction and or the presence of displacement in a fracture as the predictor for AVN. In this study, many patients had displaced fractures. The prevalence of AVN was $16.7 \%$ [8]. In contrast to this study, we found a significant relationship between fracture displacement, type of reduction, and the frequency of postoperative complications. In our research, the most important determinants of postoperative complications were the delay of surgery more than 6 hours, type of fracture, type of reduction, and mean femoral neck shortening.

\section{Study limitations}

Given the retrospective nature of the study, as well as the low frequency of reoperations, the clinical variation of patients cannot be evaluated. The main problem of this study was the frequent follow-up and persuasion of 
the patient to be responsive while adhering to the principles of research ethics. Finally, the surgeons in this study were different, which is another limitation of this study.

\section{Conclusion}

In this study, the frequency of complications was lower in patients with anatomic reduction. As a result, the anatomic reduction has a significant effect on improving the health of patients and reducing postoperative complications. More than 6 hours' delay in operation significantly increases the postoperative complications. As a result, reducing the delay in surgery in patients with FNF can reduce the occurrence of postoperative complications significantly. The type of fracture as an independent factor (that only the mechanism of trauma is involved in its development) is not modifiable and affects the frequency of complications. As a result, complications were significantly higher in type III and IV fractures.

\section{Study suggestions}

Considering the lack of descriptive studies, we propose the relevant studies with larger sample sizes. A study to examine the relationship between the types of FNF treatments, their effects, and the duration of patients' recovery with respect to the level of physical activity before the injury is recommended to identify the benefits and therapeutic potential of each treatment. In all procedures, using internal fixation, selection, and prioritizing patients for this treatment is essential. It is better the risk factors that improve or weaken the prognosis of patients' treatment to be comprehensively evaluated for selecting patients who receive the best outcome from this treatment.

\section{Ethical Considerations}

\section{Compliance with ethical guidelines}

This study began after the approval of the Ethics Committee in the Biomedical Research of Vice-Chancellor for Research and Technology of Iran University of Medical Sciences (Code: IR.IUMS.FMD.REC.1397.199). Patients' information was kept confidential. The study patients entered the study after giving their consent and could leave at each stage of the research.

\section{Funding}

The present paper was extracted from the Residency thesis of the third author Bone and Joint Reconstruction Research Center, Shafa Orthopedic Hospital, Iran University of Medical Sciences, Tehran.

\section{Authors' contributions}

Conceptualization: Karim Pisoudeh, Kaveh Gharanizadeh; Methodology, writing - original draft: Karim Pisoudeh, Mohammad Reza Sarshar; Resources, investigation: All authors; Writing - review \& editing: Mani Mahmoudi; Supervision: Karim Pisoudeh, Mani Mahmoudi.

\section{Conflict of interest}

The authors declared no conflict of interest.

\section{References}

[1] Davidovitch RI, Jordan CJ, Egol KA, Vrahas MS. Challenges in the treatment of femoral neck fractures in the nonelderly adult. J Trauma. 2010; 68(1):236-42. [DOI:10.1097/TA.0b013e3181c428ce] [PMID]

[2] Ahmadi-Abhari S, Moayyeri A, Abolhassani F. Burden of hip fracture in Iran. Calcif Tissue Int. 2007; 80(3):147-53. [DOI:10.1007/s00223-006-0242-9] [PMID]

[3] Hoskins W, Rayner J, Sheehy R, Claireaux H, Bingham $R$, Santos R, et al. The effect of patient, fracture and surgery on outcomes of high energy neck of femur fractures in patients aged 15-50. Hip Int. 2019; 29(1):77-82. [DOI:10.1177/1120700018761154] [PMID]

[4] Pavone V, Testa G, Riccioli M, Di Stefano A, Condorelli G, Sessa G. Surgical treatment with cannulated screws for pediatric femoral neck fractures: A case series. Injury. 2019; 50(Suppl. 2):S40-4. [DOI:10.1016/j.injury.2019.01.043] [PMID]

[5] Chan DS. Femoral neck fractures in young patients: State of the art. J Orthop Trauma. 2019; 33(Suppl. 1):7-11. [DOI:10.1097/BOT.0000000000001366] [PMID]

[6] Berggren M, Stenvall M, Englund U, Olofsson B, Gustafson Y. Co-morbidities, complications and causes of death among people with femoral neck fracture-a three-year follow-up study. BMC Geriatr. 2016; 16(1):120. [DOI:10.1186/ s12877-016-0291-5] [PMID] [PMCID]

[7] Marti RK, Schuller HM, Raaymakers EL. Intertrochanteric osteotomy for non-union of the femoral neck. J Bone Joint Surg Br. 1989; 71(5):782-7. [DOI:10.1302/0301620X.71B5.2584247] [PMID]

[8] Purcell RL, Cody JP, Gordon W, Kilcoyne K. Outcomes of war related femoral neck fractures. Injury. 2015; 46(12):2399-403. [DOI:10.1016/j.injury.2015.10.016] [PMID] 
This Page Intentionally Left Blank 